\title{
THE BULGE STELLAR POPULATION IN M31
}

\author{
P. JABLONKA \\ D.A.E.C, Observatoire de Paris, France \\ T. BRIDGES \\ RGO, United Kingdom \\ G. MEYLAN \\ ESO, Germany \\ AND
}

A. SARAJEDINI

KPNO, USA

We obtained WFPC2 high spatial resolution images, in filters F555W and F814W, of a few fields in the bulge of M31. Two are located SW along the major axis of M31, respectively at 6.1 and 3.2 arcmin of the galaxy nucleus; another is located $\mathrm{NE}$ along the major axis at 3.7 arcmin. Adopting $1 \mathrm{arcmin}=250 \mathrm{pc}$ from Rich \& Mighell (1995), these separations correspond to projected distances of about $1.55,0.80$, and $0.92 \mathrm{kpc}$, respectively. We present here some of our results after analysis of the $I ; V-I$ colour magnitude diagrams of the bulge stellar population, which are part of an extensive work to be published elsewhere (Jablonka et al. 1997).

An increase of RGB tip luminosity towards the center of M31 had been reported from ground-based and HST (aberrated) observations. The possible existence of either an intermediate age stellar population or a new and rare state of stellar evolution was mentioned (Mould et al. 1986 ; Mould 1986 and Rich \& Mighell 1995). Figure 1 displays these RGB tip levels in I band (crosses) as a function of the distance to the M31 center.

The observations that we present here are done in regions located at comparable radii from the galaxy nucleus as those explored in previous studies. This allows us a new investigation of earlier claims. Our determination of the upper limits to the RGB tips for the 3 different fields are shown with arrows in Figure 1. They are all much fainter than was found before. 


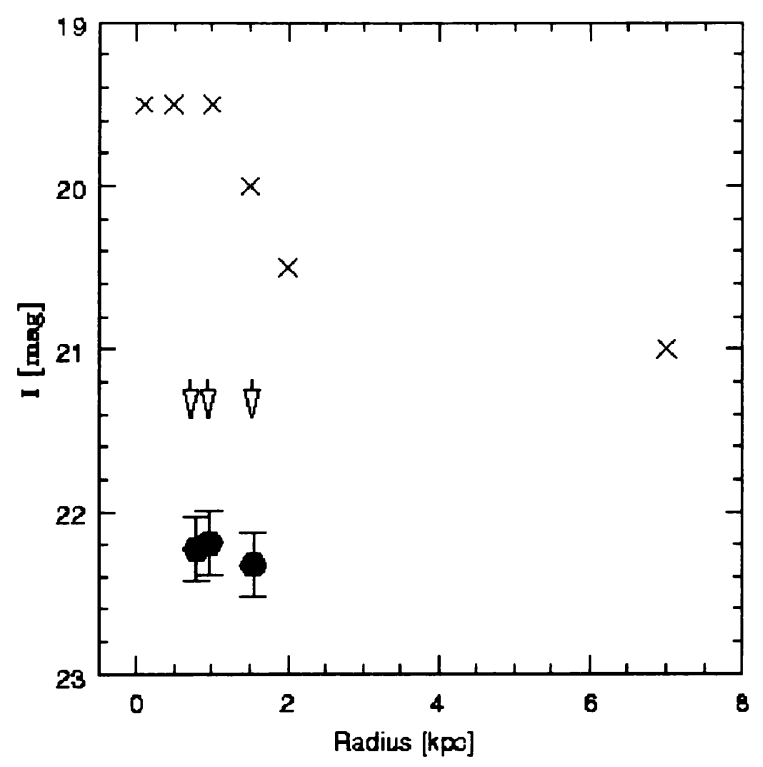

Figure 1. The I magnitude of the Red Giant Branch Tip as a function of distance to the M31 center

The high density of stars $\left(\sim 65,000\right.$ detected in a $36^{\prime \prime} \times 36^{\prime \prime}$ area $)$ likely implies that only the spatial resolution of the refurbished HST could handle such an extreme medium. The brightening encountered in previous analyses appears to have been due to blending of unresolved stars.

Dots in the figure give more precise determination of the RGB tips that we get. They correspond to what is normally expected for an old and extremely metal-rich stellar population (Bica et al. 1991). They indicate that the M31 bulge is at least as metal-rich as the bulge of our Galaxy, or more (see for comparison Rich 1996).

\section{References}

Bica E. Barbuy B., Ortolani S., ApJ, 382, L15

Jablonka P., Bridges T., Maeder A., Meylan G., Meynet G., Sarajedini A., 1997, in preparation

Mould J., Kristian J., 1986, ApJ, 305, 591

Mould J., 1986, in Stellar Populations, Eds Renzini, p.9

Rich M.R., Mighell K.J., 1995, ApJ, 439, 145

Rich M.R., 1996, in Formation of the galactic halo.. inside and out", ASP Conf. Series 92 , page 24

Simien F., de Vaucouleurs G., 1986, ApJ, 302, 564 